\title{
Abordagens de marketing e orientação para mercado aplicadas às médias empresas brasileiras
}

\author{
Marketing approaches and market orientation applied to medium-sized brazilian \\ companies
}

\author{
José G. Lupoli Junior ${ }^{1}$ \\ Yasmin Magalhães dos Santos ${ }^{2}$
}

\begin{abstract}
Resumo
A literatura de administração recomenda que, para o desenvolvimento de uma organização, é necessário aplicar abordagens estratégicas de marketing e de orientação para mercado. Apesar do crescimento vertiginoso da produção técnica, pouco se produz na área de marketing orientado para as médias empresas, um setor que, embora de grande relevância econômica, carecem de suporte técnico para se desenvolverem. Diante disso, a questão problema que emerge é: quais seriam essas carências técnicas de marketing? Responder tal pergunta foi o principal objetivo deste trabalho. Para tanto, conduziu-se um estudo exploratório qualitativo, baseado em uma amostra de 66 empresas de médio porte, que procurou identificar as necessidades estratégicas de marketing dessas organizações para desenvolver a orientação para o mercado e, consequentemente, alavancar o seu desenvolvimento. Os resultados obtidos identificaram que os principais problemas se relacionam a quatro abordagens estratégicas: Gestão Comercial, Segmentação de Mercado, Posicionamento Competitivo e Marketing de relacionamento. A pesquisa mostrou ainda pouco conhecimento sobre o potencial da aplicação de metodologias e técnicas para se obter resultados organizacionais superiores. Por fim, $\mathrm{O}$ trabalho conclui, discutindo possiveis soluções para reduzir as carências do setor.
\end{abstract}

Palavras-chave: Médias empresas. Orientação para o mercado. Competitividade. Marketing estratégico.

\begin{abstract}
The literature on business administration recommends that, for the organization development, it is necessary to apply strategic approaches on marketing and on market orientation. Although the massive growth in technical production, little is produced related to marketing addressed to medium-sized enterprises - a segment that, though having a high economic relevance, it lacks technical support to develop. Therefore, the question that emerges is: what would be these marketing technical needs? Answering this question was the primary objective of this paper and, to accomplish this objective, a qualitative exploratory research was conducted, based on a sample of 66 medium-sized enterprises. The research pursued to identify medium-sized enterprises strategic needs in marketing, to develop a program of market orientation and, therefore, improve its growth. The obtained results showed that the main problems are related to four strategic approaches: Commercial Management, Market Segmentation, Competitive Positioning and Relationship Marketing. The research also showed the little knowledge about the potential of applying methodologies and techniques to attain superior organizational results. Finally, the paper concludes discussing possible solutions to reduce the medium-sized enterprises segment needs.
\end{abstract}

Keywords: Medium Enterprises. Market Orientation. Competitiveness. Marketing strategy.

\section{Introdução}

A despeito da importância para a economia brasileira, percebe-se que médias empresas (MEs) não recebem a proporcional atenção da academia que, nitidamente, concentra sua produção para as micro, pequenas e para as grandes corporações empresariais.

Professor Doutor do Departamento de Marketing da USP. Afiliação: Escola de Artes Ciências e Humanidades da Universidade de São Paulo: EACH/USP. Brasil. Lattes: http://lattes.cnpq.br/1242550729736977 Orcid: http://orcid.org/0000-0001-5691-5232 Email: lupolijr@usp.br

2 Pesquisadora e graduanda do Curso de Marketing da Universidade de São Paulo. Afiliação: Escola de Artes Ciências e Humanidades da Universidade de São Paulo: EACH/USP. Brasil. Email: magalhaesyasmin13@gmail.com 
As micro e pequenas empresas, em razão do forte apelo social que possuem, e os grandes conglomerados, por serem referências em suas áreas de atuação, são objetos de uma extensa parte dos trabalhos acadêmicos, o mesmo não acontecendo com as médias empresas. Chetty e Campbell-Hunt (2003) reforçam a argumentação quando observam que o interesse em grandes conglomerados, como unidades de análise, domina a literatura a despeito do fato de que os pequenos e médios negócios também são importantes no processo econômico.

Pode-se sugerir que a bibliografia técnica, produzida para outros modelos organizacionais, também pode atender às demandas das médias empresas. No entanto, por suas características, suspeita-se que haja poucas oportunidades para que tais soluções sejam consideradas, sem a compreensão das suas especificidades (SIMPSON et al., 2006).

Sob o contexto do marketing, alguns estudos, realizados originalmente nos países nórdicos, têm mostrado que o conceito-chave para a sobrevivência e crescimento de empresas no atual ambiente de negócios, moldado pela complexidade e intensa competição, é o estabelecimento e manutenção de relações de troca recorrentes com os clientes no decorrer do tempo, o que só é possível quando a empresa como um todo desempenha as funções de marketing, adaptando-as às necessidades dos clientes e de potenciais clientes (GRÖNROOS, 1989; WALSH, LIMPISK, 2009; REIJONEN et al., 2012), em uma abordagem definida como "orientação para mercado" (GRÖNROOS, 1989). Kohli e Jaworski (1990) complementam a definição, acrescentando que orientação para mercado é o processo de identificação, desenvolvimento e de mobilização integrada dos recursos, conhecimentos, capacidades e competências de uma organização para entender e atender às necessidades do seu mercado/ cliente (foco no cliente), com o objetivo de lucratividade nessa relação.

Ainda sob o ponto de vista conceitual, Day (1994) introduz o conceito de "capabilidade", definindo-o como o conjunto integrado de recursos, conhecimentos e capacidades especiais que as organizações orientadas para mercado identificam e desenvolvem com o objetivo de alcançar posição de superioridade sobre concorrentes e no mercado em que atuam. Empiricamente, no entanto, percebe-se que as MEs enfrentam dificuldades para se alinharem à abordagem de orientação para mercado, já que possuem problemas específicos e característicos de gestão e de atuação no mercado (GILMORE, CARLSON e GRANT, 2001).

A tentativa de identificar tais problemas e as suas relações com a orientação para mercado é o foco deste estudo. Isto posto, a principal questão de pesquisa que o trabalho procura explorar é: quais são as principais dificuldades, relacionadas a capabilidades, enfrentadas pelas médias empresas de uma amostra, para apresentar desempenho superior em estratégias de marketing orientadas para mercado? Para tentar responder à pergunta central, o artigo em tela descreve um estudo exploratório que, por meio de entrevistas semiestruturadas com executivos e controladores de uma amostra de 66 organizações de médio porte brasileiras, objetivou identificar as principais dificuldades enfrentadas por essas organizações para atuar em seus mercados, relacionando tais dificuldades empíricas a constructos de marketing alinhados à orientação para o mercado.

Para atingir a meta principal, o estudo estabeleceu os seguintes objetivos específicos: explorar junto aos indivíduos da amostra os principais problemas, relacionados com as ações estratégicas de marketing das empresas na atuação em seus mercados; correlacionar, ordenar pela frequência e validar, por meio de entrevistas semiestruturadas com os indivíduos da amostra e de ferramentas estatísticas, os problemas identificados com abordagens e constructos de marketing; e realizar, com os indivíduos da amostra, uma análise de profundidade sobre o impacto dos quatro constructos de marketing que se mostraram mais frequentemente relacionados com os problemas estratégicos apontados.

Justifica-se este estudo, sob o ponto de vista acadêmico, pelo ainda insuficiente volume, tanto no Brasil como internacionalmente, de produções técnicas e teóricas de marketing que tenham como objeto e/ ou objetivos, especificamente, a média empresa (CHASTON, MANGLES, 2002; NASCIMENTO, TEIXEIRA, 2012). Resnick et Al. (2011), com base no contexto acadêmico britânico, reforçam o argumento quando afirmam que, embora esteja em franco crescimento, os estudos sobre pequenas e médias empresas ainda são pouco explorados no ensino e na prática do marketing. Um setor que, apesar de oferecer contribuições importantes para a economia brasileira e global, é carente de suporte técnico para o seu desenvolvimento 
e amadurecimento. A lacuna é tão grande que a imensa maioria das fontes pesquisadas para este estudo apresentou informações combinando dados de pequenas e médias organizações, sem distingui-las (PWC, 2013; IBGE, 2014; SEBRAE, 2014).

Se não bastasse a argumentação acima para justificar e salientar a relevância deste trabalho, acrescentase também seu impacto no contexto gerencial e prático dos executivos das MEs. Um estudo dedicado a pesquisar as realidades competitivas de médias empresas, a analisar as suas principais dificuldades na atuação comercial e de marketing, e a discutir possíveis soluções para seus desafios de mercado é um subsídio técnico de grande valia para gestores e controladores de médios negócios.

A seguir serão discutidos os conceitos de média empresa, estratégia de marketing e de orientação para mercado. Abordagens que fundamentaram este trabalho.

\section{Revisão Teórica}

\subsection{Conceitos de média empresa}

Segundo Oliveira Lima (2001), não existe fundamentação legal na definição de média empresa no Brasil, ao contrário das de micro/pequeno e as de grande porte, que são definidas por textos legais. Diferentes órgãos: Receita Federal, IBGE, BNDES, Sebrae e outros, utilizam diversos parâmetros para classificar as empresas no país (GUIMARÃES; CARVALHO; PAIXÃO, 2018) O Serviço Brasileiro de Apoio às Micro e Pequenas Empresas - Sebrae (2012) baseia sua classificação do porte das organizações, considerando dois critérios: número de empregados e setor de atuação: indústria, comércio e/ou serviços, para isso.

Já o Instituto Brasileiro de Geografia e Estatística - IBGE (2014) utiliza o número de funcionários sem considerar o setor de atividade da companhia para essa classificação, denominando os termos: micro, pequeno, médio e grande empreendimento apenas escalonando quatro intervalos pelo número de trabalhadores contratados: 0 a 9 pessoas (micro), de 10 a 49 pessoas (pequena), de 50 a 249 pessoas (média) e 250 pessoas ou mais (grande). Esse critério é também o mais utilizado internacionalmente, destacando-se na Comunidade Europeia (FLOYD; MCMANUS, 2005) e nos Estados Unidos da América (WALSH; LIPINSKI, 2009; REIJONEN; LAUKKANEN, 2010).

Por conveniência, este trabalho considerou a metodologia do Banco Nacional de Desenvolvimento Econômico e Social - BNDES (2015), que considera a receita anual das organizações como o critério discricionário de classificação do seu porte: microempresa -receita até $R \$ 2,4$ milhões; pequena - a partir de $R \$ 2,4$ milhões até $R \$ 16$ milhões; média - a partir de $R \$ 16$ milhões até $R \$ 90$ milhões; e grande empresa - acima de $R \$ 90$ milhões.

Dados do Sebrae (2018) dão conta que no Brasil existem, aproximadamente, 45.000 mês, que representam $0,6 \%$ do total de empresas constituídas no Brasil, ofertando aproximadamente 5 milhões de empregos e contribuindo com $15,4 \%$ das receitas geradas pelas empresas em todo o país.

Embora a maioria dos dados estatísticos e informacionais, tanto no Brasil (IBGE, 2014; PWC, 2013; PUGA, 2000) como no exterior (LAUKKANEN et al., 2013; O'DWYER; GILMORE; CARSON, 2009; MORIARTY et al., 2008; BERTHON; EWING; NAPOLI, 2008; LONGENECKER et al., 1997), misturem informações sobre as pequenas e as médias empresas sem distingui-las; as MEs diferem não apenas pelo volume de funcionários e receitas, mas também por estarem em transição de uma administração personalista, para uma gestão profissional (GABRIELSSON, 2007a; ZAHRA; NEUBAUM; HUSE, 2000).

As MEs diferem também das pequenas empresas por apresentarem maior participação do corpo gerencial profissionalizado nas decisões estratégicas. Ao contrário das últimas, que tendem a ser mais dependentes do seu fundador e/ou controlador (GABRIELSSON, 2007b). Ainda como característica diferencial, as MEs requerem maior sofisticação no planejamento e no controle da gestão, pois seus procedimentos são mais formalizados e os processos de avaliação de desempenho e recompensas são mais presentes (BENNETT e ROBSON, 2004). 


\subsection{Marketing e orientação para mercado nas médias empresas}

Moriarty et al. (2008) escrevem que há evidências fortes para se sugerir que, para ter sucesso em seus mercados de atuação, as pequenas e médias empresas necessitam dominar e aplicar abordagens de marketing. Sob tal enfoque, a literatura afirma que a função do marketing é criar e sustentar vantagens competitivas (BETTIOL; DI MARIA; FINOTTO, 2012).

De uma forma específica, o marketing visa assegurar que as capabilidades de uma organização sejam compatíveis com as evoluções do ambiente em que opera (DAY, 1994; HOOLEY; SAUNDERS; PIERCE, 2001). Sua finalidade é capacitar uma organização para se diferenciar, adaptar-se às evoluções do ambiente e dos clientes, além de desenvolver atributos críticos para sua sobrevivência (BETTIOL; DI MARIA; FINOTTO, 2011; FERRARESI et al., 2012).

Autores argumentam que a orientação para o mercado - definida por Grönroos (1989) como o direcionamento de todas as atividades organizacionais para: identificar as necessidades e os desejos dos clientes de um mercado selecionado, desenvolver soluções para satisfazer tais necessidades e entregálas de forma eficaz e mais eficientemente do que seus competidores - provê para as PMEs desempenho superior e é grande responsável pelo seu crescimento (O'CASS; NGO, 2007; WALSH; LIPINSKI, 2009; REIJONEN et al., 2012; LAUKKANEN et al., 2013). Umas das causas para essa superioridade reside no fato de que as organizações direcionadas para o mercado enfocam a obtenção de dados sobre os clientes e sobre o desempenho de competidores (LAUKKANEN et al. 2013; HUGES; LE BON; MALSHE, 2012), além de buscar as capabilidades necessárias para competir (DAY, 1994; MERRILES et al., 2011).

Identifica-se que uma empresa é orientada para o mercado quando desenvolve três fatores comportamentais: orientação para o cliente, orientação para a competição e coordenação interfuncional (BLANKSON; STOKES, 2002; HUGES; LE BON; MALSHE, 2012).

No entanto, percebe-se que as MEs possuem carências importantes, tanto no conhecimento como na aplicação da orientação para mercado (MORIARTY et al., 2008). Processos estruturados não são comuns de se observar em MEs (BLANKSON; STOKES, 2002). Isto ocorre porque, em geral, não dão prioridade para o planejamento e para a execução de marketing (BLANKSON; SOTKES, 2002; MCCARTAN-QUINN; CARSON, 2003), que são as bases da orientação para mercado (KOHLI; JAWORSKI, 1990; DAY, 1994).

Além da percebida insensibilidade de gestores para as abordagens mais formais de marketing, as MEs possuem características como: recursos limitados (dinheiro, tempo, materiais, entre outros), limitações de competência técnica e tendem a ser menos formais. Por isso, suas ações são limitadas, restritas, oportunistas e, na maioria das vezes, circunstanciais (GILMORE; CARLSON; GRANT, 2001; BJERK; HULTMAN, 2002; MCCARTAN-QUINN; CARSON, 2003; SIMPSON et al., 2006; HILLS; HULTMAN; MILES, 2008; RESNICK et al., 2011). Por outro lado, possuem algumas vantagens: são empreendedoras e flexíveis, o que permite maior adaptação para atuar em nichos de mercados (HILLS; HULTMAN; MILES, 2008; BERTHON; EWING; NAPOLI, 2008; BETTIOL; DI MARIA; FINOTTO, 2011).

Em razão de tais características, autores concordam que não há muita conformidade entre o marketing aplicado nas MEs e o da bibliografia convencional, baseada nas experiências e orientações das grandes corporações (GILMORE; CARLSON; GRANT, 2001; MORIARTY et al., 2008), o que pode explicar as resistências das MEs em acessar o suporte técnico, priorizando o empirismo (SIMPSON et al., 2006; CARSON; GILMORE, 2000).

Embora haja na literatura metodologias para dimensionar o grau de maturidade em marketing e a orientação para o mercado de uma organização (vide FERRARESI et al., 2012), este estudo procurou explorar as principais deficiências em capabilidades (DAY, 1994) necessárias para se desenvolver a orientação para mercado (GRÖNROOS, 1989), enfrentadas pelos gestores de MEs em sua atuação gerencial.

\section{Metodologia de Pesquisa}

Para atingir os objetivos de identificar os principais problemas de marketing enfrentados por uma amostra de MEs, alinhando-os com a visão de orientação para mercado, utilizou-se a pesquisa exploratória, baseada na abordagem qualitativa de obtenção dos dados. 


\subsection{Tipo, método de pesquisa e obtenção dos dados}

Estudos sobre MEs são relativamente novos na pesquisa social e há pouco conhecimento sobre essas organizações. Portanto, recomenda-se que a ênfase seja dada a pesquisas empíricas. Para isso, a abordagem qualitativa, cujo objetivo é mais explicar do que predizer o fenômeno, e mais entender as coisas do que medir, mostra-se adequada (GILMORE; CARSON; GRANT, 2001). Nemkova, Souchon e Huges (2012) compartilham do mesmo entendimento quando argumentam que: "em estudos com poucas informações disponíveis, a pesquisa exploratória é capaz de fornecer um primeiro nível de entendimento da realidade do objeto de pesquisa. Por sua vez, trabalhos exploratórios que investigam áreas pouco estudadas são melhor servidos por pesquisas qualitativas."

A abordagem qualitativa é mais flexível por oferecer a oportunidade de explorar, por meio de entrevistas semiestruturadas e de profundidade, as informações quanto à percepção e avaliação dos indivíduos sobre o problema (MCDANIEL; GATES, 2003). Acrescenta-se que a pesquisa qualitativa oferece mais segurança na interpretação dos dados, já que há a oportunidade de confirmar ou não as análises do pesquisador (MORIARTY et al., 2008), por isso, é considerada mais apropriada para os estudos de marketing (STOKES, 2000).

O método de coleta de dados baseou-se em entrevistas semiestruturadas em um processo de grupo foco, definido como um método de pesquisa qualitativa em que um moderador passa um período de tempo discutindo, com um grupo de 08 a 12 participantes, de forma profunda e a exaustão um tópico ou conceito (MCDANIEL; GATES, 2003). Os dados foram colhidos durante um período de três anos, de 2014 a 2016. O método é recomendado para se obter dados para pesquisas qualitativas de profundidade (BIASOLI ALVES; SILVA, 1992).

O roteiro orientou-se para explorar as percepções dos entrevistados sobre as principais deficiências percebidas pelos entrevistados relacionadas com a visão de orientação para o mercado (GRÖNROOS, 1989). Na sequência, discutiu-se sobre as possíveis soluções para tais problemas. A sistematização dos dados foi compartilhada com os entrevistados, objetivando promover a mútua checagem das informações e sua consistência (FERNANDES, 1991).

No estudo em tela, realizaram-se duas reuniões por empresa, com duração de 4 horas. Os grupos foram compostos de 6 e 8 executivos, eleitos entre os altos gestores das áreas de marketing, comercial, financeira e de operações, e nas duas reuniões estiveram presentes o controlador e/ou o principal executivo.

Por fim, as deficiências identificadas foram ordenadas pela frequência relativa de citações (em percentual). A consistência do ordenamento foi verificada pelo teste de hipótese "teste de proporção" que compara se as diferenças entre as frequências percentuais das citações pelos indivíduos da amostra eram significativas, para isso considerou-se a técnica mais recomendada por autores da área para o teste de diferença de proporções para variáveis discretas e curva normal com np > 5, que é o "teste Z" (HOEL, 1981; MILONE 2004). O procedimento foi realizado com o auxílio do software "MINITAB 17".

\subsection{Qualificação da amostra}

A amostra selecionada classificou-se como não probabilística e foi obtida por conveniência, portanto, não representativa da população das MEs brasileiras, limitando generalizações para esse universo (MCDANIEL; GATES, 2003; MALHOTRA, 2012). Os indivíduos da amostra fazem parte de $66 \mathrm{MEs}$, classificadas conforme o critério de faturamento anual, convencionado pelo BNDES (2015). Respeitando o critério, a média aritmética do faturamento das empresas da amostra resultou em $R \$ 38.396 .718,75$, com um desvio padrão de $R \$$ 22.010.945,48, números que confirmam que a amostra pertence ao universo das MEs.

A amostra representou treze setores da economia, conforme a Classificação Nacional de Atividades Econômicas - CNAE (IBGE, 2014), detalhados na Figura 1. A maior participação foi de empresas do setor industrial. Quanto à origem geográfica, as empresas são dos estados de Santa Catarina (44\%), São Paulo (30\%), Bahia (9\%), Mato Grosso (9\%) e Pernambuco (8\%).

Todas as empresas pesquisadas são participantes do Programa "Parceiros para a Excelência (PAEX)", patrocinado pela Fundação Dom Cabral - FDC. 
O PAEX- FDC é definido como uma parceria entre a instituição e MEsinteressadas no aprendizado e na implantação de modelos de gestão para a melhoria de resultados e aumento da competitividade, objetivos que são atingidos pela troca de experiências, na qual os participantes colocam em prática conceitos gerenciais aprendidos (FDC, 2014).

Figura 1: Número de empresas por setor de atividade econômica

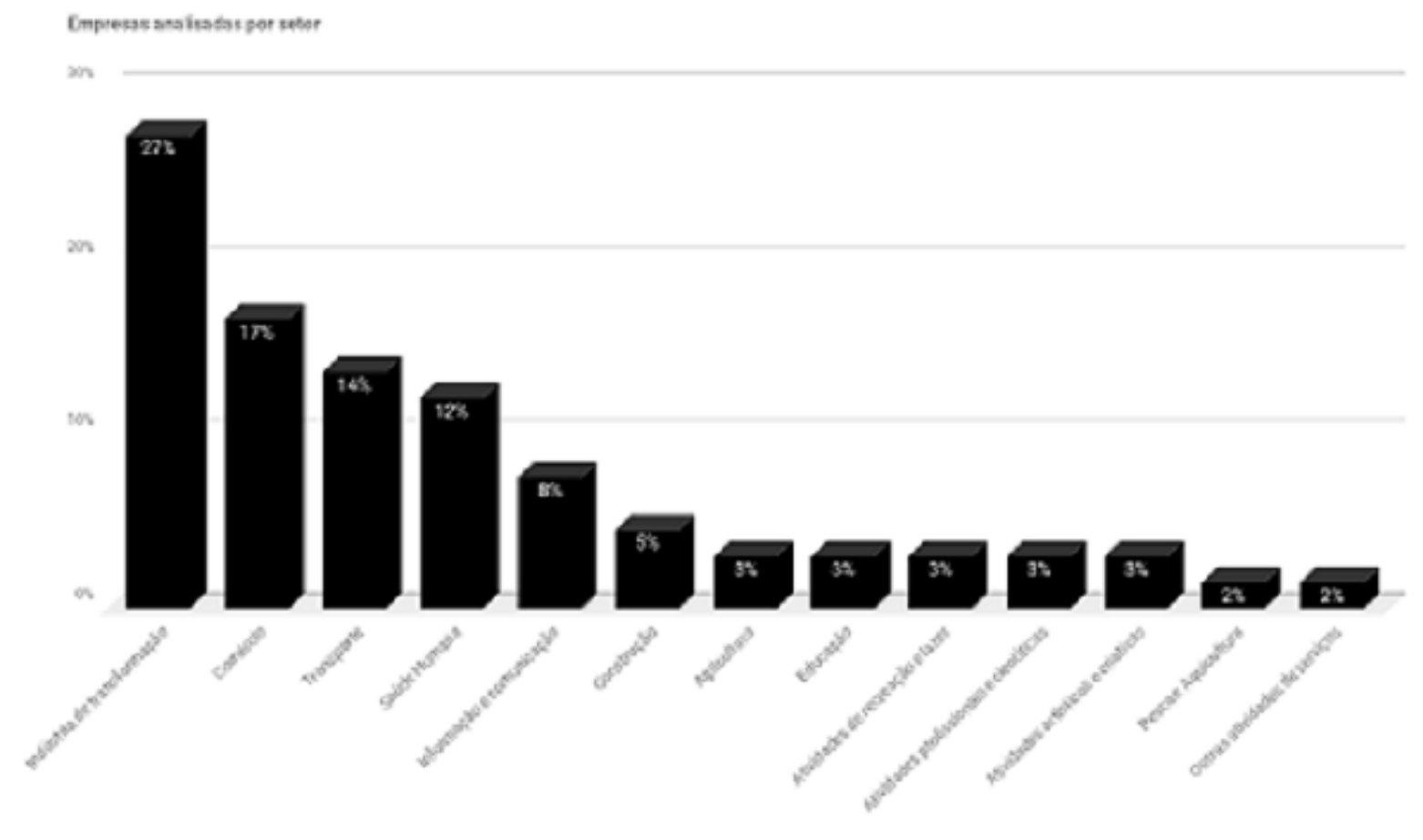

Fonte: Os autores (2017)

\section{Análise e Discussão dos Resultados}

O gráfico da Figura 2 apresenta as frequências relativas das abordagens de marketing, relacionadas diretamente com a visão de orientação para mercado (GRÖNROSS, 1989; DAY, 1994; CABRAL 2002) e deduzidas a partir das discussões com os entrevistados sobre as deficiências identificadas em suas organizações na atuação no mercado. Convencionou-se considerar como abordagens de maior frequência as que ultrapassaram o valor de dez por cento do total das citações. Por esse critério, quatro delas se destacaram: 1- Gestão comercial; 2- Segmentação de mercado; 3- Posicionamento competitivo e 4 Marketing de relacionamento.

Conforme antecipado, procurou-se avaliar o nível de confiança da ordenação, ou seja, verificar se as quatro abordagens eram proporcionalmente mais frequentes do que as demais. Considerou-se, para isso, a técnica da curva normal e "Teste Z" (MILONE, 2004).

Para o teste, considerou-se a sexta abordagem mais citada: "composto promocional $\left(P_{P}\right)$ ", como referência para as demais. Em outras palavras, se as quatro abordagens de marketing foram mais citadas do que a sexta abordagem, estariam em posição superior às outras. Citações que não apresentaram relações com o arcabouço teórico do marketing foram desconsideradas, apenas destacadas na Figura 2 como "estratégias não aplicadas a marketing".

Para a representação nos cálculos do teste $Z$, as proporções das abordagens foram denominadas: gestão comercial $\left(P_{G C}\right.$; segmentação de mercado $\left(P_{S}\right)$; posicionamento competitivo $\left(P_{C P}\right)$; e marketing de relacionamento: $\left(P_{M R}\right)$. As hipóteses levantadas para aplicação do teste $Z$, portanto, foram: 1) Ho: $P_{G C}=$ $P_{P}, H o: P_{G C}>P_{P}$; 2) Ho: $P_{S}=P_{P}, H o: P_{S}>P_{P}$; 3) Ho: $P_{P C}=P_{P}, H o: P_{P C}>P_{P}$; e 4) Ho: $P_{M R}=P_{P}, H o: P_{M R}>P_{P}$.

Considerou-se para os testes o nível de confiança de $95 \%$ e $\alpha=0,05$. Os resultados são apresentados nas Figuras 3, 4, 5 e 6. 
Figura 2: Ordenamento das abordagens de marketing por frequência

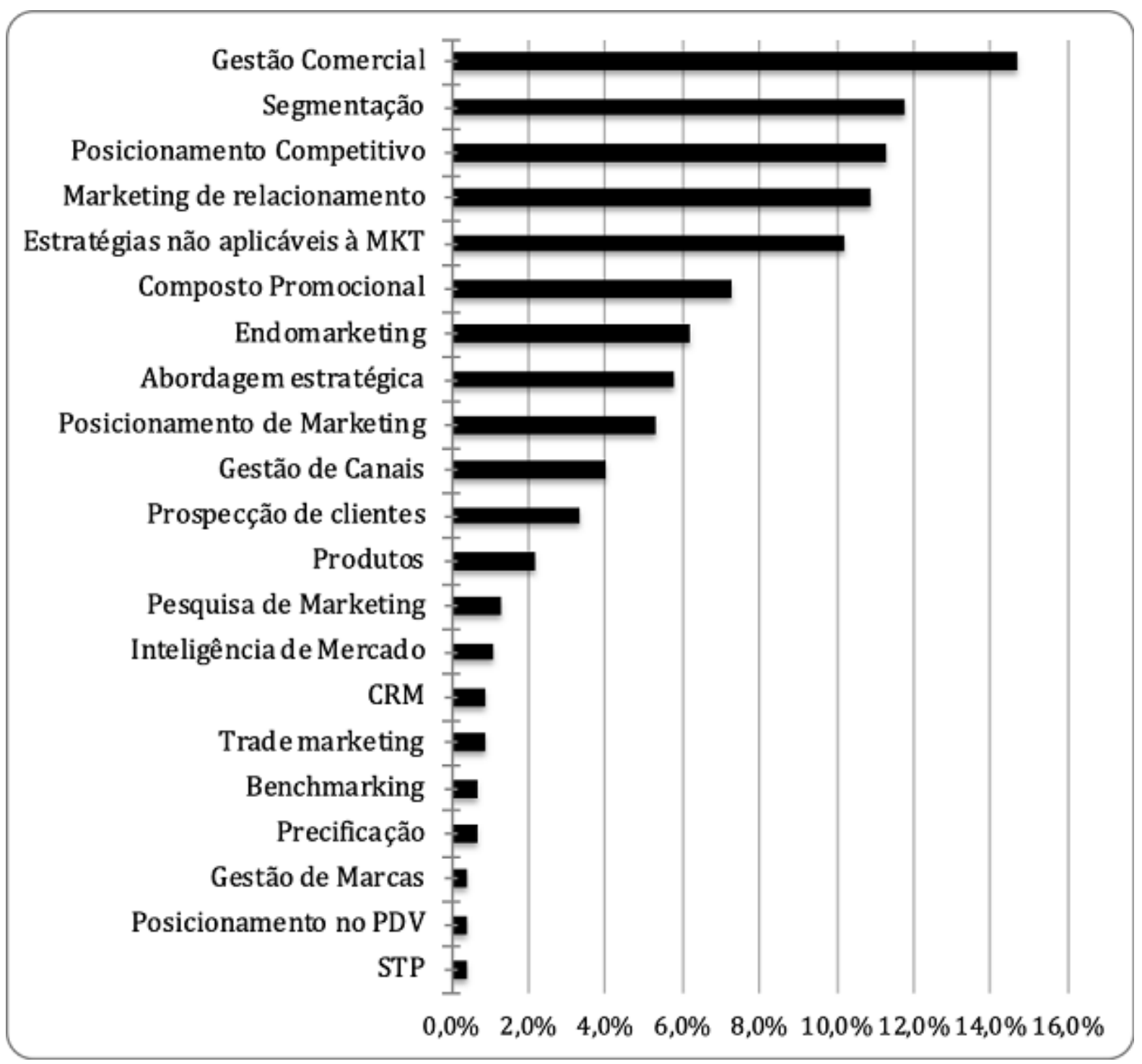

Fonte: Os autores (2017)

Figura 3: Tese z para análise de proporções, gestão comercial

Hipótese 1: Ho: $\mathrm{P}_{G C}=\mathrm{P}_{\mathrm{P}}$; Ho: $\mathrm{P}_{G C}>\mathrm{P}_{\mathrm{P}}$

\begin{tabular}{|c|c|c|c|}
\hline \multicolumn{4}{|l|}{ Teste para duas proporções } \\
\hline Sample & $\mathrm{X}$ & $\mathrm{N}$ & Sample $\mathrm{p}$ \\
\hline 1 & 66 & 450 & 0,146667 \\
\hline 2 & 33 & 450 & 0,073333 \\
\hline
\end{tabular}

Difference $=p(1)-p(2)$; Estimate for difference: 0,073333; 95\% lower bound for difference: 0,0392592; Test for difference $=0$ (vs $>0): Z=3,54$ P-value $=0,000$

Fonte: Os autores (2017)

Conclusão, considerando-se $\alpha=0,05$, existem evidências para a rejeição de Ho, Prop. Gestão Comercial, que é significativamente maior do que a Prop. Composto promocional. 
Figura 4: Teste z para análise de proporções, segmentação

Hipótese 2: Ho: $P_{S}=P_{P ;}$ Ho: $P_{S}>P_{P}$

\begin{tabular}{|c|c|c|c|}
\hline \multicolumn{4}{|l|}{ Test and Cl for Two Proportions } \\
\hline Sample & $\mathrm{X}$ & $\mathrm{N}$ & Sample $\mathrm{p}$ \\
\hline 1 & 53 & 450 & 0,117778 \\
\hline 2 & 33 & 450 & 0,073333 \\
\hline
\end{tabular}

Difference $=p(1)-p(2)$; Estimate for difference: 0,044444; 95\% lower bound for difference: 0,0122997; Test for difference $=0$ (vs $>0): Z=2,27$ P-value $=0,011$

Fonte: Os autores (2017)

Considerando $\alpha=0,05$, há evidências para a rejeição de Ho, Prop. Segmentação, que é significativamente maior do que a Prop. Composto promocional.

Figura 5: Teste z para análise de proporção, posicionamento competitivo

Hipótese 3: Ho: $\mathrm{P}_{\mathrm{PC}}=\mathrm{P}_{\mathrm{P} ;}$ Ho: $\mathrm{P}_{\mathrm{PC}}>\mathrm{P}_{\mathrm{P}}$

\begin{tabular}{|c|c|c|c|}
\hline Test and Cl for Two Proportions \\
\hline Sample & $\mathrm{X}$ & $\mathrm{N}$ & Sample $\mathrm{p}$ \\
\hline 1 & 51 & 450 & 0,113333 \\
\hline 2 & 33 & 450 & 0,073333 \\
\hline
\end{tabular}

Difference $=p(1)-p(2)$; Estimate for difference: 0,04; 95\% lower bound for deferent: 0,00817640; Test for difference $=0$ (vs > 0): $Z=2,07$ P-value $=0,019$

Fonte: Os autores (2017)

Considerando $\alpha=0,05$, há evidências para a rejeição de Ho, Prop. Posicionamento Competitivo, que é significativamente maior do que a Prop. Composto promocional.

Figura 6: Teste z para análise de proporções, marketing de relacionamento

Hipótese 4: Ho: $\mathrm{P}_{M R}=\mathrm{P}_{\mathrm{P} ;}$ Ho: $\mathrm{P}_{\mathrm{MR}}>\mathrm{P}_{\mathrm{P}}$

\begin{tabular}{|c|c|c|c|}
\hline \multicolumn{4}{|l|}{ Test and Cl for Two Proportions } \\
\hline Sample & $\mathrm{X}$ & $\mathrm{N}$ & Sample $\mathrm{p}$ \\
\hline 1 & 49 & 450 & 0,108889 \\
\hline 2 & 33 & 450 & 0,073333 \\
\hline
\end{tabular}

Difference $=p(1)-p(2)$ Estimate for difference: 0,0355556; 95\% lower bound for deferent: 0,00406018. Test for difference $=0$ (vs $>0): Z=1,86$ P-value $=0,032$

Fonte: Os autores (2017)

Considerando $\alpha=0,05$, há evidências para a rejeição de Ho, ou seja, a Prop. Marketing de Relacionamento é significativamente maior do que a Prop. do Composto Promocional.

\subsection{Análise qualitativa: principais problemas e abordagens de marketing}

As quatro abordagens de maior frequência possuem estreita ligação entre si e são consistentes com as dimensões conceituais de orientação para mercado: identificação e seleção do mercado (segmentação), desenvolvimento de soluções superiores à concorrência (posicionamento competitivo) e entrega das soluções para os clientes (gestão comercial e marketing de relacionamento) (GRÖNROOS, 1989; KOHLI; JAWORSKI, 1990; DAY, 1994; BLANKSON; STOKES, 2002; REIJONEN et al., 2012).

Não se observou na amostra planejamentos estratégicos formais de marketing, o que se mostrou coerente com a bibliografia de que as MEs são menos sensíveis às abordagens formais (GILMORE; CARLSON; GRANT, 2001; MCCARTAN-QUINN; CARLSON, 2003; SIMPSON ET AL., 2006; HILLS; HULTMAN; MILES, 2008). No entanto os achados sinalizaram que, coerente com os argumentos de Bjerk e Hultman (2002), a 
amostra se mostrou sensível à visão estratégica, visto que as abordagens mais citadas compõem, segundo Kotler e Keller (2012), a base de um plano estratégico de marketing.

Embora a bibliografia não considere vendas como parte do arcabouço conceitual do marketing (SHAPIRO, 2002), seu grande objetivo é viabilizar os planos e objetivos da estratégia de marketing. As vendas executam a estratégia (PANAGOPOULOS; AVLONITIS, 2009) e, se não estiver integrada ao marketing, o plano será falho (SHAPIRO, 2002).

Além disso, a frequência apresentada pela abordagem "marketing de relacionamento" sugere um forte alinhamento com as três primeiras, principalmente, por sua estreita relação com a "gestão comercial", já que empresas, diante da atual competição, estão migrando de uma abordagem oportunista, baseada em "forçar o cliente a comprar", para uma abordagem que privilegia o atendimento das necessidades dos clientes (ALVAREZ, 2012).

A seguir, aprofunda-se a discussão sobre cada uma das 4 abordagens mais frequentes. Para tanto, optou-se por analisar cada abordagem incluindo novos referenciais teóricos, tendo em vista que, seguindo a lógica da pesquisa, as mesmas apenas se tornaram salientes após o estudo de campo.

\subsubsection{Gestão comercial}

A quantidade dos problemas, relacionados à gestão comercial, sugere ser uma consequência da situação vivenciada pelas MEs no atual ambiente de negócios, um ambiente que exige novas formas de atuação comercial, alinhadas às evoluções do mercado, caracterizado pela intensa concorrência e pelo excesso de oferta de produtos e serviços (ALVAREZ, 2012).

Os principais problemas expostos pela amostra foram (citações acima de $20 \%$ do total): melhoria da eficiência no gerenciamento de desempenho de vendedores (40,74\%); falta de políticas comerciais $(35,18 \%)$; falta de conhecimento de vendedores em termos de: mix de produtos/serviços, público-alvo, posicionamento da empresa e diferenciais $(24,07 \%)$.

Conceitualmente, as vendas e o marketing são funções direcionadas ao mercado (MALSHE et al., 2017). A literatura considera que o marketing se orienta para a estratégia de longo prazo, já a gestão comercial é orientada para o curto prazo (HOMBURG; JENSEN, 2007). O marketing é o responsável pelo desenvolvimento da estratégia, bem como por gerenciar sua execução por meio das vendas (BIEMANS et al., 2010; MALSHE et al., 2017).

O processo estratégico de orientação para mercado e seus três principais componentes (orientação para o cliente, foco na concorrência e coordenação interfuncional) pressupõe a integração e a coordenação das áreas de marketing e vendas para se atingir os dois primeiros componentes (HUGES; LE BON; MALSHE, 2012). A ausência de cooperação entre o marketing e as vendas é apontada pela literatura como um dos principais causadores de efeitos adversos na percepção de valor da empresa e dos seus produtos/serviços pelos clientes, comprometendo, consequentemente, o desempenho nos negócios (MALSHE et. al., 2017; HUGES; LE BON; MALSHE, 2012; GUENZI; TROILO, 2007).

Se não houver a definição clara pelo marketing de parâmetros, como mercado-alvo, objetivos de mercado, a forma com que a empresa irá competir nesse mercado e as razões da compra (diferenciais), não há como estabelecer objetivos, metas e indicadores para a gestão e controle da eficácia e eficiência da atividade comercial (MALSHE et al., 2017).

As discussões mostraram, ainda, que tais preocupações eram quase inexistentes para a grande maioria das empresas da amostra. O que se observou foi que a totalidade das empresas media o desempenho da gestão comercial apenas pelo indicador volume de vendas, quantidade e/ou em valores monetários, um indicador financeiro e restrito, pouco útil para acompanhar a execução de uma estratégia orientada para mercado.

Nas entrevistas também se observou que os treinamentos de vendedores ocorriam, em sua grande maioria, no campo, baseados em procedimentos e rotinas de vendas, sem preocupações com abordagens relacionadas com: estratégias mercadológicas, público-alvo, escopo geográfico para atuação de vendas, entre outros temas estratégicos de marketing. 
Na contramão de tais necessidades, percebe-se uma carência na bibliografia de marketing que oriente essas organizações para soluções que atendam as suas necessidades. Os principais textos disponíveis no Brasil não abordam a estratégia comercial e as suas implicações com o marketing, concentrando-se em discutir temas restritos, como venda pessoal, técnicas pontuais de vendas/negociação e generalidades operacionais sobre a estrutura do setor comercial. Citam-se, por exemplo, algumas bibliografias usadas em cursos, tanto na graduação como na pós-graduação de marketing:Kotler e Keller (2012), Kerin et al. (2007), Churchill e Peter (2005), Nickels e Wood (1999), Evans e Berman (1997).

\subsubsection{Segmentação de mercado}

A segmentação é uma das mais importantes abordagens para fundamentar uma estratégia orientada para mercado (THOENI; MARSHALL; CAMPBELL, 2016; BRUNING; HU; HAO, 2009). O conceito é definido como o processo de dividir um mercado em um número mais homogêneo de submercados, denominados de segmentos, com base nas necessidades, na estrutura de compras e no comportamento de consumo (THOENI; MARSHALL; CAMPBELL, 2016). A relevância para a estratégia de marketing e, consequentemente, para uma abordagem de orientação para mercado, concentra-se no fato de que a identificação e a exploração das diferenças entre grupos qualificam as decisões e fortalecem a estratégia, por permitir um entendimento sobre as características, os interesses e as necessidades do segmento, auxiliando a organização a desenvolver soluções específicas, estruturar ações estratégicas e oferecer diferenciais competitivos (GALLAGHER; O'CONOR; GILMORE, 2016; CRAFT; HASSAN, 2015).

Especificamente para as MEs; que possuem menor capacidade e recursos se comparadas às grandes corporações (HILLS; HULTMAN; MILES, 2008; SIMPSON et al., 2006; MCCARTAN-QUINN; CARSON, 2003; GILMORE; CARLSON; GRANT, 2001), e por enfrentarem dificuldades para atuarem competitivamente, a segmentação se torna ainda mais relevante. Isto porque, entre outros benefícios, oferece a possibilidade de reduzir a dispersão de recursos materiais, humanos e de marketing, o que aumenta as probabilidades de conversão de clientes (MUHAMAD; ALWI, 2012; LUPOLI JR., 2002).

O estudo identificou que, da amostra, $12 \%$ apresentaram problemas relacionados à segmentação. Entre esses, os mais citados foram: desconhecimento da atratividade dos segmentos, não distinção entre clientes lucrativos e problemáticos $(28,8 \%)$; atuação geográfica ineficiente $(28,8 \%)$; dificuldade em reconhecer as necessidades dos clientes e criar soluções customizadas (23\%); falta de foco na prospecção de novos clientes $(19,2 \%)$.

Explorando as possíveis causas de tais problemas com os entrevistados, reforçaram-se as suspeitas de que as deficiências, relacionadas à segmentação de mercado, têm origem na carência técnica em marketing dos profissionais, e chama a atenção que muitos indivíduos mostraram-se céticos quanto à importância da segmentação para o desenvolvimento e suporte de estratégias de orientação para mercado de suas organizações, suspeitas alinhadas com os achados de Berthon, Ewing e Napoli (2008) e de Blankson e Stokes (2002).

Porém percebeu-se que a maioria dos entrevistados possui uma clara consciência das limitações dos recursos de suas empresas para atuar em mercados não segmentados. Isto ressalta a importância de se dar maior atenção para o conceito, já que uma das principais contribuições que a abordagem oferece é o de aumentar a eficiência de marketing e vendas, já que propicia melhor entendimento do mercado: de suas necessidades e comportamento, permitindo que se reduza a dispersão de recursos (VALERIU; TUDOR; ANDREI, 2011).

Os dados do estudo em tela também sinalizam que a orientação gerencial das empresas da amostra é, majoritariamente, voltada para o curto prazo, deixando, $=$ em segundo plano, $=0$ planejamento de longo prazo, orientação básica do marketing estratégico (O'DWYER et al., 2009; BLANKSON; SOTKES, 2002; MCCARTAN-QUINN; CARSON, 2003; BLANKSON; STOKES, 2002). Tal comportamento é coerente com os achados de Graham, citado por Kronmeyer e Kliemann (2005), em trabalhos com gerentes de PMEs que apontaram que a maioria renunciaria a um investimento que oferecesse retorno significativo de capital em longo prazo, se isto significasse desempenhos trimestrais ruins. 


\subsubsection{Posicionamento Estratégico}

O termo "posicionamento" é aplicado em dois sentidos: o estratégico e o de marca. O posicionamento de marca é o processo de estabelecer percepções em clientes sobre a imagem de uma marca (FUCHS; DIAMANTOPOULOS, 2010), serviços e/ou de uma corporação. Seu objetivo é direcionar as ofertas e as ações de marketing da companhia para construir uma imagem na mente do cliente (RIES; TROUT, 2009). O termo refere-se a um constructo estratégico que orienta empresas a dirigir esforços para um segmento de interesse (HASSAN; CRAFT, 2012).

O termo "posicionamento estratégico" refere-se ao processo de determinar a forma com que a empresa irá competir em determinado mercado, alinhando recursos para estabelecer vantagens e diferenciais sobre os seus concorrentes, construindo uma posição superior (DIMINGO, 1988). Relaciona-se às decisões sobre como uma empresa irá competir pela seleção dos mercados-alvo mais sensíveis aos pontos fortes da organização (HOOLEY; SAUNDERS; PIERCY, 2001).

Na contagem realizada, os problemas alinhados ao conceito somaram $11,23 \%$ do total das citações, $50 \%$ deste total relacionaram-se com a necessidade de se identificar os fatores e as competências fudamentais para promover vantagens competitivas; e $47,6 \%$ do total orientou-se para as dificuldades em comunicar e sensibilizar o mercado-alvo sobre tais vantagens.

A primeira dificuldade apontada não se relaciona apenas com o posicionamento estratégico, mas também com os problemas identificados pela amostra em relação à segmentação de mercado. A bibliografia de marketing é clara ao enfatizar a importância da relação entre a segmentação e o posicionamento no contexto da orientação para o mercado (HASSAN; CRAFT, 2012). Pesquisadores observam a grande dificuldade para uma empresa de menor porte em atender mercados diversos, principalmente por enfrentar restrições dos mais variados recursos e porque compradores possuem diferentes necessidades em termos de produtos, serviços, preços, distribuição, etc., em suas aquisições (ETZEL; WAKER; STANTON, 2007). Isto significa que, para uma empresa identificar diferenciais e/ou desenvolvê-los, é necessário respeitar a sequência lógica, recomendada pela estratégia de marketing, de primeiro conduzir um processo de segmentação de mercado para depois tomar as decisões sobre a forma com que a mesma se posicionará estrategicamente. O esforço de uma organização para criar diferenciais de concorrentes exige a seleção de grupos de clientes, seleção que irá subsidiar o processo de identificação dos pontos de diferença e de paridade da mesma em relação a sua concorrência (VALERIU; TUDOR; ANDREI, 2011).

Explorando o segundo problema, apontado pela amostra, observou-se que, na grande maioria das empresas pesquisadas, havia uma total dissociação entre os setores de marketing e comercial. Percebeuse que o marketing era visto como o promotor de eventos da empresa. Segundo um dos entrevistados, seria "o responsável pelo oba-oba na empresa"; elou como uma "agência interna de publicidade". A área comercial, por sua vez, era considerada como o principal vetor de atuação da empresa no mercado, o que pode explicar o porquê da dificuldade em comunicar os diferenciais do produto, serviços e/ou da própria empresa, já que, para tanto, há a necessidade de intensa integração entre as áreas (MALSHE et al., 2017).

Marketing e vendas são as principais funções de contato com o cliente da empresa (ROUZÉS; HULLAND, 2014). O envolvimento das duas áreas é fundamental para o desenvolvimento e a implantação de uma estratégia orientada para mercado e, consequentemente, oferecer valor superior para o cliente, fundamentos do posicionamento competitivo (DEWSNAP; JOEBBER, 2009; GUENZI; TROILO, 2007).

Toledo e Hemzo (1991) argumentam sobre o posicionamento no contexto do marketing estratégico, quando afirmam que esse, juntamente com a abordagem de segmentação de mercado, são partes integrantes do núcleo do marketing estratégico. O posicionamento é uma sequência do processo de segmentação de mercado (ROSSINI et al., 2016)

Isto posto, pode-se concluir que os problemas apresentados pelas empresas da amostra não são estanques e independentes entre si. Percebe-se claramente que, pela frequência com que os dois principais pilares da estratégia de marketing (as abordagens de segmentação e de posicionamento), aparecem na pesquisa, que uma das grandes dificuldades das empresas da amostra é dominar metodologias básicas do marketing estratégico. Essa é uma percepção que confirma os registros na literatura sobre o nível de 
conhecimento insatisfatório de profissionais de MEs (GILMORE; CARLSON; GRANT, 2001; MCCARTANQUINN; CARSON, 2003; SIMPSON et al., 2006; HILLS; HULTMAN; MILES, 2008).

\subsubsection{Marketing de relacionamento}

Embora o estudo até aqui tenha mostrado uma realidade distante da ideal, as preocupações em aprimorar o relacionamento com clientes, apontadas pela amostra como a quarta maior dificuldade, promove algum otimismo com a evolução em marketing dessas organizações.

A abordagem conceitual que propõe o estreitamento do relacionamento com os clientes é o marketing de relacionamento, um conceito definido por Berry (2002, p. 60) como: "um esforço integrado das empresas para identificar, construir e manter relacionamentos com clientes, em um processo de troca voltada para $o$ longo prazo".

A popularização da abordagem deveu-se à necessidade das empresas de enfrentar a escassez de recursos, principalmente matéria-prima, a amplitude da competição, que está atingindo níveis globais, e as exigências dos clientes em contar com parceiros que se integrem com suas "cadeias de suprimento", em detrimento de fornecedores eventuais. Tais fatores tornaram custoso o modelo de "vendas pontuais" e do alto giro de clientes (SHETH, 2002).

Vandenbosch e Dawar (2002) acrescentam que a disseminação e o barateamento do acesso à tecnologia, aliada à abertura dos mercados, têm provocado questionamentos sobre as fontes de vantagens competitivas em produtos tangíveis. A oferta de produtos de alta qualidade e com alta tecnologia é um ponto de paridade entre os concorrentes, não mais uma vantagem diferencial. Fatores como conveniência, flexibilidade, suporte de serviços e relacionamento com o fornecedor são tão ou mais importantes para compradores do que o desempenho do produto ou do serviço básico (LUPOLI JR., 2012).

Dos problemas relacionados com a abordagem de marketing de relacionamento, os mais citados foram: alto nível de infidelidade dos clientes (53,6\%); desconhecimento das necessidades e demandas dos clientes (24,4\%); e ausência de canais de comunicação com o cliente $(7,3 \%)$. Os dados mostram que, se ainda não fica clara a presença de um comportamento alinhado às atuais necessidades e exigências do ambiente de negócios, há consciência entre os entrevistados sobre a importância do marketing de relacionamento.

Segundo a maioria dos entrevistados $(62 \%$ dos que destacaram problemas relacionados com a abordagem), muitas dessas preocupações surgem pelos novos conhecimentos adquiridos como os conceitos de custos de transação e dos custeios baseados em atividades (activity-based costing - $A B C$ ), que estão permitindo dimensionar o custo de prospectar um cliente e comparar com os de se relacionar com a sua própria carteira. Isto nos leva à conclusão de algo que a bibliografia de marketing aborda há muito tempo: o quanto se gasta mais na prospecção e conversão de novos clientes se comparado à administração do relacionamento com os clientes da base da empresa (KOTLER; KELLER, 2012; SHETH, 2002).

Outro ponto saliente da pesquisa de profundidade (31\% dos entrevistados) foi a percepção de que nem todos os clientes são lucrativos, um dado que confirma as conclusões de Grönroos (2000), de que $30 \%$ dos clientes em uma empresa dão prejuízo. Os entrevistados se mostraram sensíveis à importância de se direcionarem para clientes lucrativos, realizando investimentos discricionários e valorizando aqueles que apresentam os maiores potenciais de ganhos, criando barreiras à saída e substituição, uma das principais funções do marketing de relacionamento (BERRY, 2002; VAN RAIJ; VERNOOIJ; TRIEST, 2003).

Quanto ao "desconhecimento das necessidades e interesses dos clientes", essa dificuldade possui forte relação com as discussões anteriores, principalmente com a abordagem de segmentação, à medida que, pelos custos do investimento, o marketing de relacionamento deve ser desenvolvido para segmentos específicos de clientes. Sheth (2002) recomenda segmentar em dois níveis, os clientes transacionais e os clientes relacionais (de alto potencial de lucratividade). Reijonen e Laukkanen (2010) propõem que uma estratégia de relacionamento deve se basear em informações dos clientes de maior valor, identificados por um processo de segmentação, e só depois prospectá-los e mantê-los. 


\section{Conclusões}

Não há questionamentos sobre a relevância das MEs e as suas contribuições para a economia brasileira, seja na geração de empregos, seja pela sua participação no produto interno bruto. A despeito dessa importância, há uma oferta restrita de produções acadêmicas e/ou técnicas, direcionadas para essas empresas, o que reforça a relevância de se produzir trabalhos que explorem o setor, seja como objeto, seja como objetivo de estudo.

No seu esforço para explorar as necessidades de marketing e de orientação para o mercado das MEs, o presente estudo, conduzido com uma amostra de empreendimentos de médio porte, identificou que a estratégia dessas empresas para desenvolver orientação para o mercado enfrenta problemas ligados, principalmente, a quatro constructos: gestão comercial, segmentação de mercado, posicionamento competitivo e marketing de relacionamento.

Os resultados também sugeriram que, embora haja a sensibilidade para a importância do domínio e da aplicação de abordagens estratégicas de marketing, não é algo presente e tangível para os executivos que atuam nas empresas da amostra.

Com base nos problemas apontados pela pesquisa, percebeu-se, ainda, que a carência técnica é grande em conhecimentos básicos de marketing, o que acende sinais de alerta para os setores de qualificação técnica, acadêmico e para os profissionais dessas organizações.

Isto posto, o estudo chama a atenção da pesquisa e da produção acadêmicas de marketing para maior mobilização dos pesquisadores no sentido de minimizar as carências do setor.

Embora o caráter exploratório limite a extensão e a generalização dos resultados aqui encontrados, as contribuições deste trabalho, como base para estudos futuros, são bem tangíveis. Sob o contexto da pesquisa, caberiam pesquisas quantitativas que confirmassem, ou não, se há relação dos principais problemas das MEs para desenvolver a orientação para mercado com os quatro constructos de marketing, aqui estabelecida. Também seriam importantes futuros estudos que pudessem contribuir com os executivos das MEs, preparando-os e capacitando-os para enfrentar as dificuldades gerenciais do atual ambiente de negócios, o que, sem dúvida, seria de grande relevância para as MEs na busca por maior competitividade.

\section{Referências}

ALVAREZ, F. J. Administração estratégica de vendas. In: OLIVEIRA, B. (org.) Gestão de Marketing. São Paulo: Pearson, 2012.

BENNETT, T.; ROBSON, P. The composition of boards of directors and strategic control: effects on corporate strategy. Academy of Management Review, v. 15, n. 1, p. 72-80, 2004.

BERRY, L. Marketing of services perspectives from 1983 and 2000. Journal of Relationship Marketing, V. 1, n. 1, p. 59-77, jan. 2002.

BERTHON, P.; EWING, M.; NAPOLI, J. Brand Management in Small to Medium-Sized Enterprises. Journal of Small Business management, v. 46, n. 01, p. 27-45, 2008.

BETTIOL, M.; DI MARIA, E.; FINOTTO, V. Marketing in SME: the role of entrepreneurial sense making. International Entrepreneurship and Management Journal, v. 1, n. 8, p. 223-248, feb. 2012.

BIASOLI ALVES, Z.; SILVA, M. Análise qualitativa de dados de entrevista: uma proposta. Pandéia (Ribeirão Preto), Ribeirão Preto, v. 1, n. 2, fev/jul. 1992.

BIEMANS, W.; MALKOVEC BRENCIC, M.; MALSHE, A. Marketing-sales interface configurations in B2B firms. Industrial Marketing Management, v. 39, n. 2, p. 183-194, 2010.

BNDES. Apoio às micro, pequenas e médias empresa, 2015. Disponível em: <https://web.bndes.gov. br/bib/jspui/bitstream/1408/4261/1/Cartilha\%20MPME\%202015.pdf>Acesso em: 15 mai. 2017.

BJERK, B.; HULTMAN, C. Entrepreneurial marketing the growth of the small firms in the new economic era. Cheltenham UK: Edward Elgar, 2002. 
BLANKSON, C.; STOKES, D. Marketing practices in the UK small business sector. Marketing Intelligence \& Planning, v. 20, n. 1, p. 49-61, 2002.

BRUNING, E.; HU, M.; HAO, W. Cross-national segmentation: an application of the NAFTA airline passenger Market. European Journal of Marketing, v. 43, n. 11/12, p. 1498-1522, 2009.

CABRAL, J. E. Orientação para o mercado e performance na indústria de alimentos: estado da arte. Revista de Ciências Administrativas, v.8, n.1, 2002.

CARSON, D.; GILMORE, A. SME Marketing Management Competencies. International Business Review, v. 9, n.3, p. 363-382, 2000.

CHASTON, I.; MANGLES, T. Small Business Marketing Management, Basingstoke: Palgrave Publishers, 2002.

CHETTY, S.; CAMPBEL-HUTT, C. Paths to internationalization among small-to medium-sized firms: a global versus regional approach. European Journal of Marketing, v. 37, n. 5/6, p. 796-820, 2003. Disponível em: <https://doi.org/10.1108/03090560310465152> Acesso em: 24 set. 2017.

CHURCHILL, G.; PETER, J. P. Marketing: criando valor para os clientes. São Paulo: Saraiva, 2005.

CRAFT, F.; HASSAN, S. Global consumer Market segmentation strategy decisions and managerial assessment performance. In: SPOTTS, H. (ed.) Revolution in Marketing: Marketing Driving Changes. Manchester: Springer International Publishing, 2015.

DAY, G. The capabilities of market-driven organizations. Journal of Marketing, v. 58, n.4, p. 37- 52, October 1994.

DEWSNAP, B.; JOBBER, D. An exploratory study of sales-marketing integrative devices. European Journal of Marketing, v. 43, n. 7/8, p. 985-1007, 2009.

DIMINGO, E. The fine art of positioning. Journal of Business Strategy, p. 34-38, mar/apr. 1988.

ETZEL, M.; WAKER, B.; STANTON, W. Marketing. 14. ed. New York: McGraw-Hill/Irwin, 2007.

EVANS, J.; BERMAN, B. Marketing. 7. ed. New York: Prentice-Hall, 1997.

FLOYD, D.; McMANUS, J. The role of SMEs in improving the competitive position of the European Union. European Business Review, v. 17, n. 2, p.144-155, 2005.

FDC, Fundação Dom Cabral. Parceiros para a excelência (PAEX). Disponível em: <https://www.fdc.org. br/hotsites/mail/paex/2014/hotsite_encontro/pt/paex.html> Acesso em: 20 fev. 2017.

FERNANDES, M. Memória Camponesa. Anais da $21^{a}$ Reunião Anual de Psicologia, SPRP, Ribeirão Preto, 20 pags.

FERRARESI, A.; DOS SANTOS, S.; FREGA, J.; PEREIRA, H. knowledge management, market orientation, innovations and organizational outcomes: a study on companies operating in Brazil. Revista de Gestão da Tecnologia e Sistemas de Informação, v. 9, n. 1, p. 89-108, jan/abr. 2012.

FUCHS, C.; DIAMANTOPULOS, A. Evaluating the effectiveness of brand-positioning strategies from a consumer perspective. European Journal of Marketing, v. 44, n. 11/12, p. 1763-1786, 2010.

GABRIELSSON, J. Boards of directors and entrepreneurial posture in medium-size companies.

International Small Business Journal, v. 25, n. 5, p. 511-537, 2007 a.

Correlates of board empowerment in small companies. Entrepreneurship Theory and Practice, v. 31, n. 5, p. 687-711, 2007.

GALLAGHER, D.; O'CONNOR, C.; GILMORE, A. An exploratory examination of the strategic direction of the Gaelic Athletic Association via the application of sports marketing segmentation bases. Marketing Intelligence \& Planning, v. 34, n. 2, p. 203-222, 2016.

GILMORE, A.; CARLSON, D.; GRANT, K. SME marketing in practice. Marketing Intelligence \& Planning, v. 19, n. 1, p. 6-11, 2001. 
GRÖNROOS, C. Defining Marketing: a market-oriented approach. European Journal of Marketing, v. 23, issue 1, p. 52-60, 1989.

GRÖNROOS, C. Service Management and Marketing: A customer relationship management approach. Chichester: Wiley, 2000.

GUENZI, P.; TROILO, G. The joint contribution of marketing and sales to the creation of superior customer value. Journal of Business Research, v. 60, n. 2, p. 98-107, 2007.

GUIMARÃES, A.; CARVALHO, K.; PAIXÃO, L. Micro, pequenas e médias empresas: conceitos e estatísticas. Radar, n. 55, fev 2018. Disponivel em: http://www.ipea.gov.br/portal/images/stories/PDFs/ radar/180209_radar_55_cap04.pdf, acesso em 06 mai. 2018.

HASSAN, S.; CRAFT, S. Examining world market segmentation and brand positioning strategies. Journal of Consumer Marketing, v. 29, n. 5, p. 344-356, 2012.

HILLS, G.; HULTMAN, C.; MILES, M. The evolution and development of entrepreneurial marketing. Journal of Small Management, v. 46, n. 01, p. 99-112, 2008.

HOEL, P. Estatística Elementar. São Paulo: Atlas, 1981.

HOOLEY, G.; SAUNDERS, J.; PIERCY, N. Estratégia de Marketing e Posicionamento Competitivo. São Paulo: Prentice Hall, 2001.

HOMBURG, C.; JENSEN, O. Worlds of Marketing and Sales: witch differences make a difference. Journal of Marketing, v. 71, n. 3, p. 124-142, jul. 2007.

HUGES, D.; LE BON, J.; MALSHE, A. The marketing-sales interface at the interface: creating Marketbased capability. Journal of Personal Selling and Sales Management, v. 32, n. 1, p. 57-72, Winter 2012.

IBGE. Estatística do cadastro central de empresa 2014. Disponível em: <http://biblioteca.ibge.gov.br/ visualizacao/livros/liv97205.pdf> Acesso em: 15 mai. 2017.

KERIN, R; HARTLEY, S.; BERKOWITZ, E.; RUDELIUS, W. Marketing. 8. ed. São Paulo: McGrawHill, 2007.

KOHLI, A.; JAWORSKI, B. Market Orientation: the construct, research propositions, and managerial implications. Journal of Marketing, v. 54, n.2, p. 1-18, apr. 1990.

KOTLER, P.; KELLER, K. Administração de Marketing. 14. ed. São Paulo: Pearson, 2012.

KRONMEYER FILHO, R.; KLIEMANN NETO, F. Horizontes de curto, médio e longo prazo no processo de desdobramento e gestão da estratégia: Uma abordagem integradora. In: XXV ENEGEP. Porto Alegre, 2005.

LAUKKANEN, T.; NAGY, G.; HIRVONEN, S. REIJONEN, H.; PASANEN, M. The effect of strategic orientations on business performance in SMEs: a multigroup analysis comparing Hungary and Finland. International Marketing Review, v. 30, n. 6, p. 510-535, 2013.

LONGENECKER, J. C., MOORE, C. W., PALICH, L. E.; PETTY, J. W. Administração de Pequenas Empresas. São Paulo: Makron Books, 1997.

LUPOLI JR., J. Uma visão estratégica da segmentação de mercado aplicada ao Business to Business. Pensamento e Realidade, São Paulo, v. 5, n. 10, p. 45- 61, 2002.

LUPOLI JR. J. Marketing Estratégico. In DE ANGELO, C. NIELSEN, F. FOUTO, N. Manual de Varejo no Brasil. São Paulo: Saint Paul, 2012. Cap. 4, p. 75-94.

MALHOTRA, N. Pesquisa de Marketing: uma orientação aplicada. 6. ed. São Paulo: Bookman, 2012.

MALSHE, A.; FRIEND, S.; AL-KATHIB, J.; AL-HABIB, M. Strategic and operational alignment of salesmarketing interfaces: dual paths within a SME configuration. Industrial Marketing Management, v. 66, p. $145-158$, oct. 2017. 
McCARTAN-QUINN, D.; CARSON, D. Issues which Impact upon marketing in the Small Firm. Small Business Economics, Jordanstown, v. 21, p. 201-213, 2003.

MERRILES, B.; RUNDLE-THIELE, S.; LYE, A. Marketing capabilities: antecedents and implications for B2B SME performance. Industrial Marketing Management, v. 40, n. 3, p. 368-375, 2011.

McDANIEL, C.; GATES, R. Pesquisa de Marketing. São Paulo: Thomson, 2003.

MILONE, G. Estatística Geral e Aplicada. São Paulo: Thompson Learning, 2004.

MORIARTY, J.; JONES, R.; ROWLEY, J.; KUPIEC-TEAHAN, B. Marketing in small hotel: a qualitative study. Marketing \& Planning, v. 26, n. 3, p. 293-315, 2008.

MUHAMAD, R.; ALWI, S. Segmentation and brand positioning for Islamic financial services. European Journal of Marketing, v. 46, n. 7/8, p. 900-921, 2012.

NASCIMENTO, M.; TEIXEIRA, J. O marketing na literatura versus o marketing nas pequenas empresas: concepções de empresários de pequenas empresas de um município de Minas Gerais. Revista Eletrônica de Estratégia \& Negócios, v. 5, n. 2, p. 229-256, 2012.

NEMKOVA, E.; SOUCHON, A.; HUGES, P. Export decision-making orientation: an exploratory study. International Marketing Review, v. 29, n. 4, p. 349-378, 2012.

NICKELS, W.; WOOD, M. Marketing: relacionamentos, qualidade, valor. Rio de Janeiro: LTC, 1999.

O'CASS, A.; NGO, L. Market orientation versus innovative culture: two routes to superior brand performance. European Journal of Marketing, v. 41, n. 7/8, p. 868- 887, 2007.

O'DWYER, M.; GILMORE, A.; CARSON, D. Innovative marketing in SME: a theoretical framework. European Business Review, v. 21, n. 6, p. 504-515, 2009.

OLIVEIRA LIMA, E. As definições de Micro, Pequena e Média Empresas Brasileiras como Base para a Formulação de Políticas Públicas. In: Anais do II EGEPE, Londrina, PR, 2001. p. 421-436.

PANAGOPOULOS, N. AVLONITIS, G. Performance implications of sales strategy: the moderating effects of leadership and environment. International Journal of Research in Marketing, v. 27, n. 1, p 46-57, nov. 2009.

PUGA, F. P. Experiências de Apoio às Micro, Pequenas e Médias Empresas nos Estados Unidos, na Itália e em Taiwan. In: Textos para Discussão 75 - BNDES. Rio de Janeiro, 2000. Disponível em: www. bndes.gov.br Acesso: 24 mai. 2016.

PWC, Price Watherhouse Coopers. Pequenas e Médias Empresas: Private Company Services. 2013. Disponível em: <http://www.pwc.com.br/pt/publicacoes/setores-atividade/assets/pcs/private-compay> Acesso em: 24 mai. 2016.

RIES, AL; TROUT, J. Posicionamento: a Batalha pela sua Mente. São Paulo: M. Books, 2009.

REIJONEN, H.; LAUKKANEN, T. Customer relationship oriented marketing practices in SMEs. Marketing Intelligence \& Planning, v. 28, n. 2, p. 115-136, 2010.

REIJONEN, H.; LAUKKANEN, T.; KOMPPULA, R.; TUOMINEN, S. Are growing SMEs more Marketoriented and brand-oriented? Journal of Small Business Management, v. 50, n. 4, p. 699-716, 2012.

RESNICK, S.; CHENG, R.; BRINDLEY, C.; FOSTER, C. Aligning teaching and practice: a study of SME marketing. Journal of Research in Marketing and entrepreneurship, v. 13, n. 1, p. 37-46, 2011.

ROSSINI, F.; MANGINI, E.; URDAN, A.; SANTOS, A. Reposicionamento de marca em pequeno varejista do setor calçadista. Revista de Ciências Administrativas, v. 22, n. 2, p. 542-572, jul./dez. 2016.

Disponível em: http://periodicos.unifor.br/rca/article/view/4157/pdf. Acesso em: 18 abr. 2018.

ROUZÉS, D.; HULLAND, J. Does marketing and sales integration Always pay off? Evidence from a social capital perspective. Journal of the Academy of Marketing Science, v. 42, n. 5, p. 511-527, 2014. 
SEBRAE. Anuário do Trabalho na Micro e Pequena Empresa 2012. Disponível em: <sebrae.com.br/ Sebrae/Portal\%20Sebrae/Anexos/Anuario\%20do\%20Trabalho\%20Na\%20Micro\%20e\%20Pequena\%20 Empresa_2012.pdf $>$. Acesso em: 15 mai. 2017.

SEBRAE. Participação das Micro, Pequenas e Médias Empresas na Economia 2014. Disponível em: http://www.sebrae.com.br/Sebrae/Portal\%20Sebrae/Estudos\%20e\%20Pesquisas/Participacao\%20 das\%20micro\%20e\%20pequenas\%20empresas.pdf. Acesso em: 24 set. 2017.

SEBRAE. How Many Companies Are There in Brazil. Disponível em: http://thebrazilbusiness.com/ article/how-many-companies-are-there-in-brazil , acesso em: 20 jul. 2018.

SHAPIRO, B. Want a happy customer? Coordinate sales and marketing. Research \& Ideas. 2002. Disponível em: <http://hbswk.hbs.edu/item/want-a-happy-customer-coordinate-sales-and-marketing> Acesso em: 30 jun. 2017.

SHETH, J. The future of relationship marketing. Journal of Service Marketing, v. 16, n. 7, p. 590-592, 2002.

SIMPSON, M.; PADMORE, J.; TAYLOR, N.; FREECKNALL-HUGES, J. Marketing in small and medium enterprises. International Journal of Entrepreneurial Behavior \& Research, v. 12, n. 6, p. 361-387, 2006.

STOKES, D. Entrepreneurial Marketing: a conceptualization from qualitative research. Qualitative Market Research: An International Journal, v. 3, n. 1, p. 47-54, 2000.

THOENI, A.; MARSHALL, G.; CAMPBELL, S. A resource-advantage theory typology of strategic segmentation. European Journal of Marketing, v. 50, n.12, p. 2192-2215, 2016.

TOLEDO, G.; HEMZO, M. O processo de posicionamento e o marketing estratégico. In: XV Encontro Anual do ANPAD. Anais, v. 4, 1991.

VALERIU, C.; TUDOR, I.; ANDREI, P. The premises of strategic marketing planning implementation within small and medium sized enterprises. Annals of the University of Oradea: Economic Science, $v$. 20, n. 1, p. 730-735, 01 jul. 2011.

VAN RAIJ, E.; VERNOOIJ, M.; TRIEST, S. The implementation of customer profitability analysis: a case study. Industrial Marketing Management, v. 32, n. 7, p. 573-583, 2003.

VANDENBOSCH, M.; DAWAR, N. Beyond better products: capturing value in customer interactions. MIT Sloan Management Review, v. 43, n. 4, p. 35- 42, summer 2002.

WALSH, M.; LIPINSKI, J. The role of marketing function in small and medium sized enterprises. Journal of Small Business and Enterprise Development, v. 16, n. 4, p. 569-585, 2009.

ZAHARA, S.; NEUBAUM, D.; HUSE, M. Entrepreneurship in medium-size companies: exploring the effects of ownership and governance systems. Journal of Management, v. 26, n. 5, p. 947-976, 2000.

Submetido em: $17 / 04 / 2019$

Aceito em: 16/06/2019 